\title{
Twin-donor systems for resonance energy transfer
}

\author{
Robert D. Jenkins ${ }^{1}$, David L. Andrews * \\ School of Chemical Sciences, University of East Anglia, Norwich NR4 7TJ, UK
}

Received 11 November 1998

\begin{abstract}
Resonance energy transfer involving two identical donors and one acceptor is modelled by quantum electrodynamical calculation. The results account for recent experimental observations of simultaneous excitation energy transfer in the photochemistry of organic dyes. It is shown that a family of three energy transfer mechanisms has to be considered in order to describe fully the energy pooling phenomenon. The chromophore architecture has a pivotal role in determining the dominant mechanism. (C) 1999 Elsevier Science B.V. All rights reserved.
\end{abstract}

\section{Introduction}

When the absorption of light leads to electronic excitation in condensed phase matter, the associated energy is, in many cases, rapidly conveyed from the site of its initial deposition to another. This process is familiar in many areas of photophysics, and its distance dependence is increasingly proving of value for the detailed analysis of intersite distances in macromolecules or proteins [1,2]. In the case of energy transfer between chemically distinct donor and acceptor species separated beyond significant wavefunction overlap, energy migration is generally considered as involving one of two mechanisms: at short distances, radiationless transfer [3] with an

\footnotetext{
* Corresponding author. E-mail: d.1.andrews@uea.ac.uk

${ }^{1}$ E-mail: robert.jenkins@uea.ac.uk
}

inverse sixth power dependence on the donor-acceptor separation - and at longer distances, radiative transfer identifiable with the well-known inverse square law. The comparatively recent development of a unified theory [4] for resonance energy transfer (RET), based on quantum electrodynamics (QED), has identified these two mechanisms as the long- and short-range limits of a more general encompassing mechanism. With due regard to the effects of the intervening medium $[5,6]$, the unified theory accommodates both intermolecular and inter-chromophore excitation transfer.

Recent studies have revealed the occurrence in certain systems of a fundamentally new three-centre process, first termed simultaneous excitation energy transfer (SEET) [7,8]. This involves the transfer of energy from two electronically excited donors to a single acceptor - the initial excitation of each donor having occurred through normal single-photon absorption. One such unimolecular system, recently 
characterised, comprises building blocks of fluorescein or erythrosin as donors, transferring energy to an $E$-stilbene acceptor unit [9]. Here the acceptor and donors are linked by thiourea units which, in themselves, inhibit through-bond relaxation and conjugated charge-delocalisation. The two donors are pre-excited using laser light at a frequency off-resonant with respect to direct excitation of the acceptor, and the observed intramolecular energy transfer is characterised by the $E / Z$-isomerisation of the stilbene $[9,10]$.

In this Letter we formulate theory for a general case of twin-donor resonance energy transfer, following the initial excitation of two chemically identical donor molecules in the vicinity of the acceptor. To be of chemical interest, the structure of the energy levels for the donor and acceptor species must dictate that: (1) the energetics preclude acceptor excitation through energy transfer from a single donor; and (2) the transfer of energy is a concerted, not a trivial stepwise process, since the latter offers nothing distinctive. As such, the process with which we are concerned is best represented by the chemical equation:

$\mathrm{A}^{*}+\mathrm{B}+\mathrm{A}^{*} \rightarrow \mathrm{A}+\mathrm{B}^{*}+\mathrm{A}$.

It is assumed that the vibronic level of each electronically excited donor $\mathrm{A}^{*}$, from which energy transfers, is a state populated by intramolecular vibrational relaxation, within the excited state manifold, prior to the interaction we here consider. The total energy transferred to the acceptor B is thus in general less than the sum of the initial donor excitation energies.

\section{QED formalism}

The Hamiltonian, $H$, for a system comprising two chemically identical donor molecules ( $\mathrm{A}$ and $\mathrm{A}^{\prime}$ ) and one acceptor $\mathrm{B}$ can be written as:

$H=H_{\mathrm{mol}}^{\mathrm{A}}+H_{\mathrm{mol}}^{\mathrm{B}}+H_{\mathrm{mol}}^{\mathrm{A}^{\prime}}+H_{\mathrm{int}}^{\mathrm{A}}+H_{\mathrm{int}}^{\mathrm{B}}+H_{\mathrm{int}}^{\mathrm{A}^{\prime}}+H_{\mathrm{rad}}$,

with $H_{\text {mol }}^{\xi}$ the Hamiltonian for molecule $\xi, H_{\text {rad }}$ the second-quantised radiation field Hamiltonian and $H_{\text {int }}^{\xi}$ the molecule-field coupling. In the electric dipole approximation the latter operator is given by:

$H_{\text {int }}^{\xi}=-\varepsilon_{0}^{-1} \boldsymbol{\mu}(\xi) \cdot \boldsymbol{d}^{\perp}\left(\boldsymbol{R}_{\xi}\right)$,

with $\boldsymbol{\mu}(\xi)$ the electric dipole moment operator and $\boldsymbol{R}_{\xi}$ the position vector of molecule $\xi$. The transverse electric displacement field operator, $\boldsymbol{d}^{\perp}\left(\boldsymbol{R}_{\xi}\right)$, can be expressed in the usual expansion in terms of radiation modes characterised by wave-vector $\boldsymbol{p}$ and polarisation $\lambda$ :

$$
\begin{aligned}
d^{\perp}\left(\boldsymbol{R}_{\xi}\right)= & \sum_{p, \lambda}\left(\frac{\hbar c p \varepsilon_{0}}{2 V}\right)^{1 / 2} \mathrm{i}\left[\boldsymbol{e}^{(\lambda)}(\boldsymbol{p}) a^{(\lambda)}(\boldsymbol{p}) \mathrm{e}^{\mathrm{i} \boldsymbol{p} \cdot \boldsymbol{R}_{\xi}}\right. \\
& \left.-\overline{\boldsymbol{e}}^{(\lambda)}(\boldsymbol{p}) a^{\dagger(\lambda)}(\boldsymbol{p}) \mathrm{e}^{\mathrm{i} \boldsymbol{p} \cdot \boldsymbol{R}_{\xi}}\right]
\end{aligned}
$$

where $\overline{\boldsymbol{e}}$ represents the polarisation vector and $\overline{\boldsymbol{e}}$ its complex conjugate, $a$ and $a^{\dagger}$ are the annihilation and creation operators, respectively, and $V$ is the quantisation volume.

In the two-donor energy pooling processes the donors $\mathrm{A}$ and $\mathrm{A}^{\prime}$ progress from an already excited state $\left|\mathrm{A}_{\alpha}\right\rangle$ to the electronic ground state $\left|\mathrm{A}_{0}\right\rangle$, and the acceptor molecule (B) is excited from its ground state $\left|B_{0}\right\rangle$ to an excited state $\left|B_{\beta}\right\rangle$, through transfer of the excitation energies of the donors $A$ and $A^{\prime}$. The energetics satisfy the basic relation $2 E_{\alpha 0}^{\mathrm{A}}=E_{\beta 0}^{\mathrm{B}}$, where $E_{\alpha 0}^{\mathrm{A}}$ is the energy lost by a donor and $E_{\beta 0}^{\mathrm{B}}$ that gained by the acceptor. In quantum electrodynamical terms the energy migrates due to the creation and annihilation of virtual photons, entailing a sum over all possible radiation modes and polarisations. The initial and final states are given by:

$$
\begin{array}{ll}
|i\rangle=\left|\mathrm{A}_{\alpha} ; \mathrm{A}_{\alpha}^{\prime} ; \mathrm{B}_{0}\right\rangle|0\rangle ; & E_{i}=2 E_{\alpha}^{\mathrm{A}}+E_{0}^{\mathrm{B}}, \\
|f\rangle=\left|\mathrm{A}_{0} ; \mathrm{A}_{0}^{\prime} ; \mathrm{B}_{\beta}\right\rangle|0\rangle ; & E_{f}=2 E_{0}^{\mathrm{A}}+E_{\beta}^{\mathrm{B}},
\end{array}
$$

where $E_{n}$ is the energy of system basis state $|n\rangle$, of the form $|n\rangle=\left|\mathrm{A}_{n} ; \mathrm{A}_{n}^{\prime} ; \mathrm{B}_{n}\right\rangle\left|\operatorname{rad}_{n}\right\rangle$. For convenience we also define $2 E_{\alpha 0}^{\mathrm{A}}=E_{\beta 0}^{\mathrm{B}}=2 \hbar c k$, the initial excitation energy of each donor represented as $\hbar c k$. The radiation ket $|0\rangle$, included for completeness, is used to signify the vacuum state where no photons are present.

The quantum probability amplitude or matrix element, $M_{f i}$, connecting the initial, $|i\rangle$, to the final, $|f\rangle$, system state is given by the lowest-order nonzero term in the time-dependent perturbation expansion - which is of fourth order since the processes 
entail two virtual photon creation and two annihilation events each:

$$
M_{f i}=\sum_{r, s, t} \frac{\left\langle f\left|H_{\mathrm{int}}\right| t\right\rangle\left\langle t\left|H_{\mathrm{int}}\right| s\right\rangle\left\langle s\left|H_{\mathrm{int}}\right| r\right\rangle\left\langle r\left|H_{\mathrm{int}}\right| i\right\rangle}{\left(E_{i}-E_{r}\right)\left(E_{i}-E_{s}\right)\left(E_{i}-E_{t}\right)} .
$$

Here we can recognise three mechanisms comprising a family of interaction pathways for energy pooling, the essential energetics of which are illustrated in Fig. 1. We have one cooperative mechanism [11]

(a)

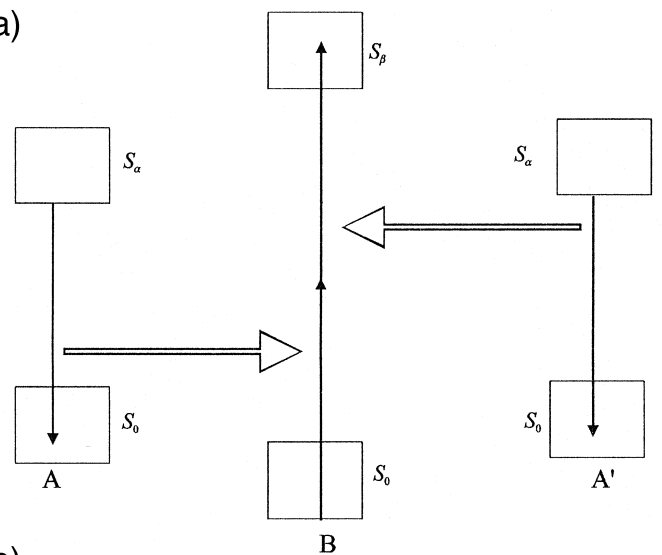

(b)

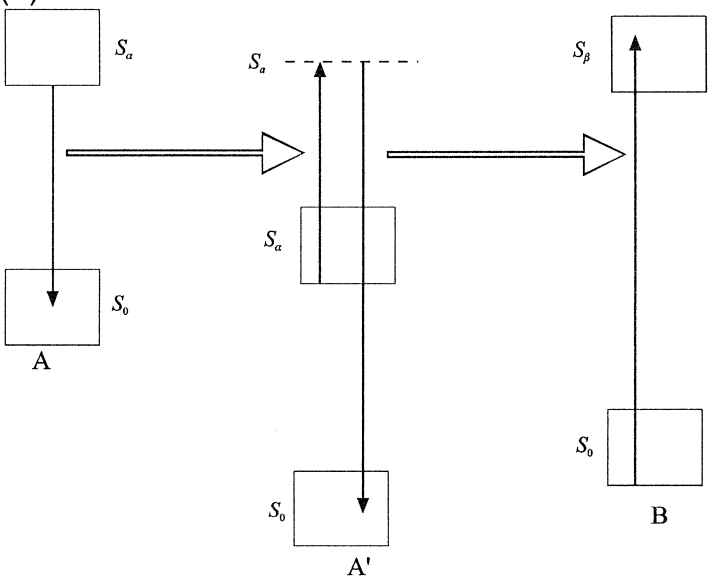

Fig. 1. Modified Jablonski diagrams showing the essential energetics of the twin-donor energy transfer processes: (a) cooperative and (b) accretive via $\mathrm{A}^{\prime}$. Here $\mathrm{S}_{0}$ represents a ground electronic state and its associated manifold; $\mathrm{S}_{\beta}$ ( or $\mathrm{S}_{\alpha}$ ) denotes a higher electronic excited state manifold; $S_{a}$ represents a virtual molecular state. Vertical arrows represent transitions; horizontal arrows denote channels of excitation. whereby the two donors transfer their excitation energy directly to the acceptor $\mathrm{B}$, thus initiating its excitation through a virtual molecular state. Along with this route we can identify two accretive energy transfer pathways where the initial excitation energy of one donor is passed to its partner and the sum of the two excitations is transferred to the acceptor. Here it is the intermediary donor which undergoes its decay transition through a virtual molecular state. In the following the notation 'ACC1' and 'ACC2' pertains to the cases where the donors $\mathrm{A}^{\prime}$ and $\mathrm{A}$, respectively, play the intermediary role.

Accommodating all possible time-orderings in (4) leads to three matrix elements, one for each of the various transfer pathways. The result for the cooperative mechanism is shown in $(5 \mathrm{a})$, whilst results for the accretive mechanisms are given in $(5 b)$ and $(5 c)$ :

$$
\begin{aligned}
& M_{f i}^{(\mathrm{COOP})}=\frac{\mu_{i}^{0 \alpha(\mathrm{A})} \mu_{l}^{0 \alpha\left(\mathrm{A}^{\prime}\right)} \alpha_{j k}^{\beta 0(\mathrm{~B})}(-k,-k)}{4 \varepsilon_{0}^{2} V^{2}} \\
& \times \sum_{p, \lambda, p^{\prime}, \lambda^{\prime}} p p^{\prime}\left\{\frac{\bar{e}_{i} e_{j} \mathrm{e}^{\mathrm{i} \boldsymbol{p} \cdot \boldsymbol{R}} \overline{\boldsymbol{e}}_{k}^{\prime} e_{l}^{\prime} \mathrm{e}^{-\mathrm{i} \boldsymbol{p}^{\prime} \cdot \boldsymbol{R}^{\prime}}}{(k-p)\left(-k-p^{\prime}\right)}\right. \\
& +\frac{\bar{e}_{i} e_{j} \mathrm{e}^{\mathrm{i} \boldsymbol{p} \cdot \boldsymbol{R}} e_{k}^{\prime} \bar{e}_{l}^{\prime} \mathrm{e}^{i \boldsymbol{p}^{\prime} \cdot \boldsymbol{R}^{\prime}}}{(k-p)\left(k-p^{\prime}\right)} \\
& +\frac{e_{i} \bar{e}_{j} \mathrm{e}^{-\mathrm{i} \boldsymbol{p} \cdot \boldsymbol{R}} \bar{e}_{k}^{\prime} e_{l}^{\prime} \mathrm{e}^{-\mathrm{i} \boldsymbol{p}^{\prime} \cdot \boldsymbol{R}^{\prime}}}{(k+p)\left(k+p^{\prime}\right)} \\
& \left.+\frac{e_{i} \bar{e}_{j} \mathrm{e}^{-\mathrm{i} \boldsymbol{p} \cdot \boldsymbol{R}} e_{k}^{\prime} \overline{\boldsymbol{e}}_{l}^{\prime} \mathrm{e}^{i \boldsymbol{p}^{\prime} \cdot \boldsymbol{R}^{\prime}}}{(-k-p)\left(k-p^{\prime}\right)}\right\}, \\
& M_{f i}^{(\mathrm{ACC} 1)}=\frac{\mu_{i}^{0 \alpha(\mathrm{A})} \mu_{l}^{\beta 0(\mathrm{~B})} \alpha_{j k}^{0 \alpha\left(\mathrm{A}^{\prime}\right)}(2 k,-k)}{4 \varepsilon_{0}^{2} V^{2}} \\
& \times \sum_{p, \lambda, p^{\prime}, \lambda^{\prime}} p p^{\prime}\left\{\frac{\bar{e}_{i} e_{j} \mathrm{e}^{\mathrm{i} \boldsymbol{p} \cdot \boldsymbol{R}^{\prime \prime}} \bar{e}_{k}^{\prime} \boldsymbol{e}_{l}^{\prime} \mathrm{e}^{\mathrm{i} \boldsymbol{p}^{\prime} \cdot \boldsymbol{R}^{\prime}}}{(k-p)\left(2 k-p^{\prime}\right)}\right. \\
& +\frac{\bar{e}_{i} e_{j} \mathrm{e}^{\mathrm{i} \boldsymbol{p} \cdot \boldsymbol{R}^{\prime \prime}} e_{k}^{\prime} \bar{e}_{l}^{\prime} \mathrm{e}^{-\mathrm{i} \boldsymbol{p}^{\prime} \cdot \boldsymbol{R}^{\prime}}}{(k-p)\left(-2 k-p^{\prime}\right)} \\
& +\frac{e_{i} \bar{e}_{j} \mathrm{e}^{-\mathrm{i} \boldsymbol{p} \cdot \boldsymbol{R}^{\prime \prime}} \bar{e}_{k}^{\prime} e_{l}^{\prime} \mathrm{e}^{\mathrm{i} \boldsymbol{p}^{\prime} \cdot \boldsymbol{R}^{\prime}}}{(-k-p)\left(2 k-p^{\prime}\right)} \\
& \left.+\frac{e_{i} \bar{e}_{j} \mathrm{e}^{-\mathrm{i} \boldsymbol{p} \cdot \boldsymbol{R}^{\prime \prime}} e_{k}^{\prime} \bar{e}_{l}^{\prime} \mathrm{e}^{-\mathrm{i} \boldsymbol{p}^{\prime} \cdot \boldsymbol{R}^{\prime}}}{(-k-p)\left(-2 k-p^{\prime}\right)}\right\} \text {, }
\end{aligned}
$$




$$
\begin{aligned}
M_{f i}^{(\mathrm{ACC} 2)}= & \frac{\mu_{i}^{0 \alpha\left(\mathrm{A}^{\prime}\right)} \mu_{l}^{\beta 0(\mathrm{~B})} \alpha_{j k}^{0 \alpha(\mathrm{A})}(2 k,-k)}{4 \varepsilon_{0}^{2} V^{2}} \\
& \times \sum_{p, \lambda, p^{\prime}, \lambda^{\prime}} p p^{\prime}\left\{\frac{\bar{e}_{i} e_{j} \mathrm{e}^{-\mathrm{i} \boldsymbol{p} \cdot \boldsymbol{R}^{\prime \prime}} \bar{e}_{k}^{\prime} e_{l}^{\prime} \mathrm{e}^{\mathrm{i} \boldsymbol{p}^{\prime} \cdot \boldsymbol{R}}}{(k-p)\left(2 k-p^{\prime}\right)}\right. \\
& +\frac{\bar{e}_{i} e_{j} \mathrm{e}^{-\mathrm{i} \boldsymbol{p} \cdot \boldsymbol{R}^{\prime \prime}} e_{k}^{\prime} \bar{e}_{l}^{\prime} \mathrm{e}^{-\mathrm{i} \boldsymbol{p}^{\prime} \cdot \boldsymbol{R}}}{(k-p)\left(-2 k-p^{\prime}\right)} \\
& +\frac{e_{i} \bar{e}_{j} \mathrm{e}^{\mathrm{i} \boldsymbol{p} \cdot \boldsymbol{R}^{\prime \prime}} \bar{e}_{k}^{\prime} e_{l}^{\prime} \mathrm{e}^{\mathrm{i} \boldsymbol{p}^{\prime} \cdot \boldsymbol{R}}}{(-k-p)\left(2 k-p^{\prime}\right)} \\
& \left.+\frac{e_{i} \bar{e}_{j} \mathrm{e}^{\mathrm{i} \boldsymbol{i} \cdot \boldsymbol{R}^{\prime \prime}} e_{k}^{\prime} \overline{\boldsymbol{e}}_{l}^{\prime} \mathrm{e}^{-i \boldsymbol{p}^{\prime} \cdot \boldsymbol{R}}}{(-k-p)\left(2 k-p^{\prime}\right)}\right\}
\end{aligned}
$$

with summation over repeated Cartesian indices implied. Here $\mu^{x y(\xi)}=\left\langle x\left|\mu^{(\xi)}\right| y\right\rangle, e_{n}$ and $e_{n}^{\prime}$ denote polarisation components of the virtual photons $(p, \lambda)$ and $\left(\boldsymbol{p}^{\prime}, \lambda^{\prime}\right)$ respectively, and the donor-acceptor separation vectors are defined as $\boldsymbol{R}=\boldsymbol{R}_{\mathrm{B}}-\boldsymbol{R}_{\mathrm{A}}, \boldsymbol{R}^{\prime}$ $=\boldsymbol{R}_{\mathrm{B}}-\boldsymbol{R}_{\mathrm{A}^{\prime}}$, and $\boldsymbol{R}^{\prime \prime}=\boldsymbol{R}_{\mathrm{A}^{\prime}}-\boldsymbol{R}_{\mathrm{A}}$. The use of prime labels on virtual photon parameters hereafter only serves to distinguish between them, for calculational convenience. The expressions of (5) are cast in terms of polarisability tensors, $\alpha^{\mathrm{fi}(\xi)}\left(k_{1}, k_{2}\right)$, of the general form [12]:

$$
\begin{aligned}
& \alpha_{j k}^{f i(\xi)}\left(\mp k_{1}, \mp k_{2}\right) \\
& \quad=\sum_{\zeta}\left\{\frac{\mu_{j}^{f \zeta(\xi)} \mu_{k}^{\zeta i(\xi)}}{\tilde{E}_{i \zeta} \pm \hbar c k_{1}}+\frac{\mu_{k}^{f \zeta(\xi)} \mu_{j}^{\zeta i(\xi)}}{\tilde{E}_{i \zeta} \pm \hbar c k_{2}}\right\},
\end{aligned}
$$

where $\zeta$ is the virtual state through which molecule $\xi$ progresses and a tilde is introduced to imply complex molecular energies. Explicitly, $\tilde{E}_{i \zeta}=E_{i \zeta}+$ i $\Gamma_{\zeta}$ with $\Gamma_{\zeta}$ the damping factor associated with state $\zeta$ and its sign fully consistent with time-reversal arguments [13]. Utilising standard methodology [12], and following a considerable amount of algebra, we can write for the completed matrix elements:

$$
\begin{aligned}
M_{f i}^{(\mathrm{COOP})}= & \mu_{i}^{0 \alpha(\mathrm{A})} V_{i j}(k, \boldsymbol{R}) \alpha_{j k}^{\beta 0(\mathrm{~B})}(-k,-k) \\
& \times V_{k l}\left(k, \boldsymbol{R}^{\prime}\right) \mu_{l}^{0 \alpha\left(\mathrm{A}^{\prime}\right)}, \\
M_{f i}^{(\mathrm{ACC} 1)}= & \mu_{i}^{0 \alpha(\mathrm{A})} V_{i j}\left(k, \boldsymbol{R}^{\prime \prime}\right) \alpha_{j k}^{0 \alpha\left(\mathrm{A}^{\prime}\right)}(2 k,-k) \\
& \times V_{k l}\left(2 k, \boldsymbol{R}^{\prime}\right) \mu_{l}^{\beta 0(\mathrm{~B})}, \\
M_{f i}^{(\mathrm{ACC} 2)}= & \mu_{i}^{0 \alpha\left(\mathrm{A}^{\prime}\right)} V_{i j}\left(k, \boldsymbol{R}^{\prime \prime}\right) \alpha_{j k}^{0 \alpha(\mathrm{A})}(2 k,-k) \\
& \times V_{k l}(2 k, \boldsymbol{R}) \mu_{l}^{\beta 0(\mathrm{~B})} .
\end{aligned}
$$

Each result is cast in terms of a second-rank, indexsymmetric, Cartesian tensor representing the retarded resonance electric dipole-electric dipole coupling of explicit form [14]:

$$
\begin{aligned}
V_{i j}(k, \boldsymbol{R})= & \frac{\mathrm{e}^{\mathrm{i} k R}}{4 \pi \varepsilon_{0} R^{3}}\left[(\mathrm{i} k R-1)\left(\delta_{i j}-3 \hat{R}_{i} \hat{R}_{j}\right)\right. \\
& \left.-k^{2} R^{2}\left(\delta_{i j}-\hat{R}_{i} \hat{R}_{j}\right)\right] .
\end{aligned}
$$

In the short-range, this coupling displays an $R^{-3}$ distance dependence, though the terms linear and quadratic in $k R$ increasingly modify the behaviour as $R$ increases. The term 'short-range' in practice indicates intermolecular separations below $R \approx 100 \AA$ (i.e., small compared to the characteristic optical distance $k^{-1}$ ), where the radiationless limit of energy transfer dominates. As $R$ increases, retardation effects $[15,4]$ become more prominent, bringing a radiative character of the transfer to the fore. Here, however, we are primarily interested in the shortrange, where the coupling exerts its greatest influence.

The rate of twin-donor energy pooling, $\Gamma$, can be ascertained by substitution of the matrix elements of (7) into the Fermi golden rule:

$\Gamma=\frac{2 \pi}{\hbar}\left|M_{f i}^{(\mathrm{COOP})}+M_{f i}^{(\mathrm{ACC} 1)}+M_{f i}^{(\mathrm{ACC} 2)}\right|^{2} \rho_{\beta}$,

where $\rho_{\beta}$ is the appropriate density of final molecular states for the acceptor, as determined by its vibronic structure. The total rate thus encompasses 'diagonal' terms associated with each of the three energy migration pathways, and also 'cross-terms' resulting from their quantum interference.

Use of the Fermi rule enables us to compare typical rates of energy pooling with the rates of RET more familiar in other systems. In conventional two-

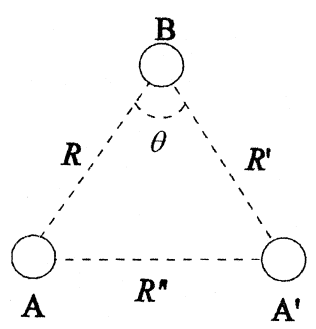

Fig. 2. Three-centre geometry and the mechanism-determining angle, $\theta$. 
body (single donor) RET, the matrix element exhibits a well-known dependence on the square of the transition dipole moments for donor and acceptor, and an $r^{3}$ dependence on intermolecular separation (with $r$ indicative of a typical chromophore separation) [4]. Each of the separate matrix elements for twin-donor energy pooling, given by (7), yields a dependence on the square of two transition dipole moments, a linear dependence on a two-photon absorption tensor and an $r^{-6}$ distance dependence. Considering a representative generalised molecular polarisablility with components of the order of $1 \times$ $10^{-39} \mathrm{C}^{2} \mathrm{~m}^{2} \mathrm{~J}^{-1}$, then for any transfer distance of a few angström it is clear that the two-donor process should offer rates comparable to conventional donor-acceptor transfer.

\section{Molecular architecture}

In an assembly of donors and acceptors grouped in twin donor/single acceptor triads, we assume that

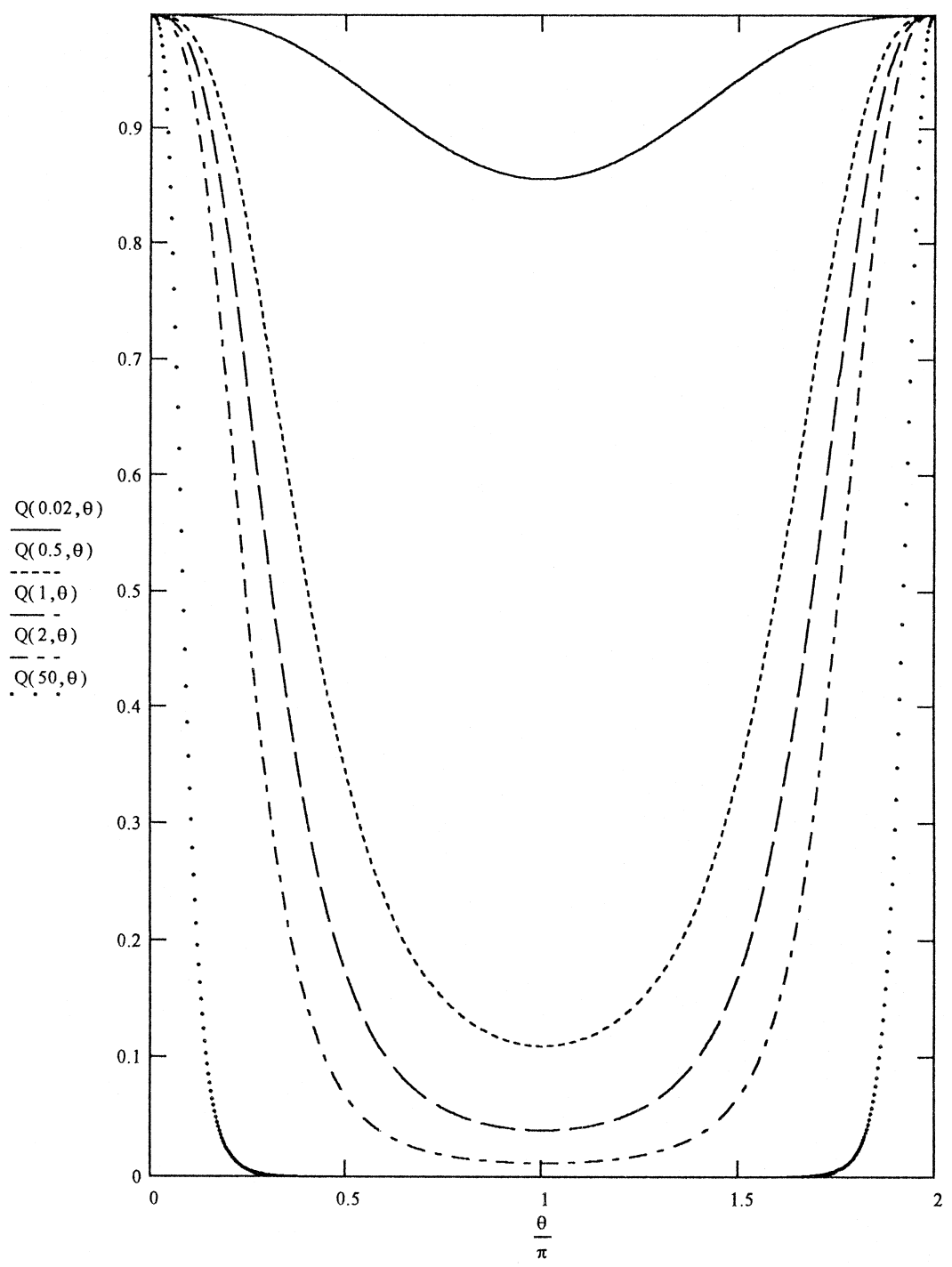

Fig. 3. Variation of the relevance function, $Q(k, \theta)$, with $\theta$. 
energy pooling within each triad is the dominant pathway for acceptor excitation, though our theory can also address inter-triad transfer. In SEET donor excitation is usually harvested at an acceptor within a single molecular triad. Specifically, we can then investigate the relative importance of competing intramolecular mechanisms on twin-donor energy transfer by considering the molecular geometry shown in Fig. 2, where $\theta$ is the angle subtended at B.

Assuming that both donors are chemically equivalent and equidistant from the acceptor we can describe the inter-donor separation $R^{\prime \prime}$ in terms of the donor-acceptor distance $R$ :

$R^{\prime \prime}=2 R \sin \left(\frac{\theta}{2}\right)$.

If we further assume the short-range form of coupling, a function relating to the relevance of the accretive mechanisms, $Q(k, \theta)$, can be evaluated:

$Q(k, \theta)=\frac{\Gamma_{\mathrm{ACC}}}{\Gamma_{\text {тОт }}}=\left\{1+4 m\left|\sin ^{3}\left(\frac{\theta}{2}\right)\right|\right\}^{-2}$,

where $\Gamma_{\text {TOт }}$ is given by (9), $\Gamma_{\mathrm{ACC}}$ is the rate of the accretive mechanisms and $m$ is related to the molecular properties of the donors and acceptor. Fig. 3 shows how the $Q$ function varies with $\theta$. A large $m$ coefficient is indicative of molecule $\mathrm{B}$ having a high polarisability which, in turn, favours the cooperative mechanism - as illustrated in Fig. 3 by the sharply decreasing trend in importance of the accretive mechanism. Conversely a low value for $m$ relates to a high polarisability for the donors, in which case the accretive mechanism becomes dominant.

Within the framework of molecular quantum electrodynamics we have shown that molecular triads exhibiting twin-donor energy pooling do so via three intertwined mechanistic pathways. Nonetheless, the interplay between the relative polarisabilities of the acceptor and donor, together with the detailed molecular architecture of the triad, exert a powerful influence in determining the dominant mechanistic pathway. Within SEET triads the central stilbene's large polarisability leads us to believe that cooperative transfer is mainly responsible for the observed $E-Z$ isomerisation.

\section{Acknowledgements}

RDJ gratefully acknowledges a research studentship funded by the Engineering and Physical Sciences Research Council.

\section{References}

[1] L. Stryer, Annu. Rev. Biochem. 47 (1978) 819.

[2] C.G. Dos Remedios, P.D.J. Moens, J. Struct. Biol. 115 (1995) 175.

[3] T. Förster, Naturwissenschaften 33 (1946) 166.

[4] D.L. Andrews, Chem. Phys. 135 (1989) 195.

[5] G. Juzeliūnas, D.L. Andrews, Phys. Rev. B 49 (1994) 8751.

[6] G. Juzeliūnas, D.L. Andrews, Phys. Rev. B 50 (1994) 13371.

[7] J. Bendig, E. Bellmann, S.J. Helm, J. Inf. Rec. Mater. 21 (1994) 441.

[8] M. Nickoleit, S. Hecht, A. Uhl, J. Bendig, J. Inf. Rec. Mater. 23 (1996) 66.

[9] M. Nickoleit, A. Uhl, J. Bendig, Laser Chem. 17 (1997) 161.

[10] Y.P. Sun, J. Saltiel, J. Phys. Chem. 93 (1989) 8310.

[11] R.D. Jenkins, D.L. Andrews, J. Phys. Chem. (in press).

[12] D.P. Craig, T. Thirunamachandran, Molecular Quantum Electrodynamics, Academic Press, New York, 1984.

[13] D.L. Andrews, S. Naguleswaran, G.E. Stedman, Phys. Rev A 57 (1996) 4925.

[14] E.A. Power, T. Thirunamachandran, Phys. Rev. A 28 (1983) 2671.

[15] L. Gomberoff, E.A. Power, Proc. Phys. Soc., London 88 (1966) 281. 